\title{
A Cytological Study of Cat Sweat Glands
}

\author{
By \\ Sadayoshi Kamamura \\ Department of Anatomy, School of Medicine, Keio-Gijuku University, Tokyo.
}

(Director: Prof. Dr. T. Taniguchi)

It $\mathrm{O}$ and his collaborators (I to et $\mathrm{O}$ is $\mathrm{hi}: 1948$, I to et I washige: 1951) have shown that the epithelium which forms the glandular tubules of the human eccrine sweat glands, is composed of cells of two different types, and these cells are generally arranged in two layers. I to named these cells the superficial and the basal cells respectively. The superficial cells are columnar epithelial cells, which surround directly the glandular cavity and the basal ends of these cells reach the membrana propria or the smooth muscle cells of the glandular tubules, but the basal cells, which are situated between the superficial cells and the membrana propria, do not reach the glandular cavity and look like parietal cells of the stomach. Comparing the nature of the secretory granules, the Golgi apparatus and the glycogen contents, I to and his collaborator concluded from the marked differences that there are two different types of glandular cells. Thereafter Tsukagoshi (1951) studied cytologically sole eccrine sweat glands of dog and cat, observing mitochondria and secretory granules, Golgi apparatus, the quantity of glycogen contents and the appocrine secretion of the superficial cells, and he concluded from his findings, that the mammalian eccrine sweat glands are also composed of cells of two different types. More recently, S c helle y et M e s c on (1952) studied the human eccrine sweat gland histochemically to demonstrate the difference between the active and the inactive glands by means of the acid and the alkaline phosphatase reactions and the glycogen stain, and also to confirm the histo- and the cytochemical changes of the glandular cells after cerspiration which is caused by the application of heat or pilocarpine injection. In the present study the author attempted to investigate this problem by observing the acid and the alkaline phosphatase activities, the amount of protein and glycogen 
contents and other cytological findings in the plantar sweat glands of the cat; and at the same time, the cytological changes in the glandular cells after the application of pilocarpine injection were observed.

\section{Material and Methods}

As the material for the study, plantar skin was taken from ten apparently healthy cats, half of which was taken without any previous treatment and another half of which was taken after a sufficient perspiration for five minutes which had been caused by the application of pilocarpine injection. The tissue was immediately put into the respective fixative solutions. The acid and the alkaline phosphatase activities were demonstrated with the procedures Gomori revised method (1952). Protein was stained with $\mathrm{Y}$ a s u ma et I c hi kawa's Ninhydrin-Sch if f's (1951), on the sections fixed with 70\% alcohol. Glycogen was stained with the periodic acid-S $\mathrm{c} h$ if $\mathrm{f}^{\prime} \mathrm{s}$ method after being fixed with $70 \%$ alcohol, and was ascertained by the diastase test. After removing paraffin the sections were treated with the $1 \%$ aqueous solution of diastase at $37^{\circ} \mathrm{C}$ for 24 hours, and when the colour reaction disappeared the coloured substance was recognized as glycogein. For further cytological investigations, the sections, which were fixed with Levi's solution, were stained with $\mathrm{Heidenhain's} \mathrm{iron}$ hematoxylin. All material was embedded in parrffin, and $5 \mu$ thick sections were cut. The sections from the pilocarpine injected material and the non-injected material were taken in pairs, and stained under the same conditions for the comparative ovservations.

\section{Original Findings}

1. acid phosphatase

a. Findings in the case without the pilocarpine injection (Fig. 1)

In a glandular cell, the most intense activity is seen on the nucleus, and the nuclear membrane shows an especially strong reaction. Being covered by the general deep coloration of the whole nucleus the existence of the nucleolus is not conspicuous though it shows also an intense activity. Next to the nucleus, the cytoplasm shows a considerably intense coloration, especially the part near the glandular cavity gives a strong reaction. Tunica propria and smooth muscle cells show little activity, and the majority of them looks transparent. The arrangement and the shape of the glandular cells of the cat 
eccrine sweat glands are like the human glandular cells irregular and the boundary lines between cells are not clear enough to be traced completely. Therefore it is impossible to catch the exact outline of these cells. In general, however, the glandular cells are the mixture of cylindrical, cubic and polygonal cells. The nuclei of the glandular cells are generally large and round or ellipsoid in shape, and their arrangement is also irregular. It is clearly seen in most cases that the glands are composed of two different types. The cells of one type face the glandular cavity and show a strong enzyme activity; the cells of another type do not reach the glandular cavity and give a weak activity. These findings agree with the result obtained by I t 0 , who observed by means of the iron hematoxylin stain technique. The cells of the former type are the superficial cells and the cells of the latter type are the basal cells. In the cytoplasm of the superficial cells, there are granules which are probably mitochondria and give a relatively strong activity. Among these granules there are somewhat larger granules with a stronger activity and are mostly found in the upper and the lateral portion of the nucleus.

The basal cells show a far weaker activity than that of the former cells. The perinuclear portion of the cytoplasm gives a com. paratively intense enzyme activity. The granules and the threads, which are considered to be mitochondria, also show a positive reaction. The threads, however, are few in number and run parallel with the basal face. A few large granules, which give a strong activity, are also found. The socalled secretory vacuoles are hardly seen. Rarely do protuberances of the glandular cells project into the glandular cavity. This picture means probably the appocrine secretion of the superficial cells, which was described by Ito et Tsukagoshi. The difference of the intensities of the enzyme activity between the nuclei of the superficial and the basal cells is not distinct.

b. Findings in the case with the pilocarpine injection (Fig. 2)

No change is observed in the tunica propria, in smooth muscle fibers or in the nuclei. The glandular cells give generally a somewhat intenser activity. The activity in the cytoplasm of the superficial cells becomes stronger than what is shown before the injection, and the granules increase in number. The basal cells give distinctly a strong activity, being stained dark as a whole. The activity is intense in the perinuclear regions, especially in their basal portions. The thread-like or granular bodies, which are considered as mitochondria, both show an intensified activity (Fig. 11). 
2. Alkaline phosphatase

a. Findings in the case without the pilocarpine injection (Fig. 3). The most intense activity is seen in the tunica propria and in the smooth muscle fibers. The intensity of glandular cells comes to the next. The borderlines between glandular cells are mostly indistinct. Some of the nuclei show a strong activity and the other of them give a weak activity, and the latter are seen more often than the former. Nucleoli are hardly visible. The intensity of the enzyme activity in the cytoplasm of glandular cells is irregular, and the borderlines between cells are mostly not distinct enough to distinguish each individual cell. The intensity of the activity in the cytoplasm, however, is generally stronger in the basal part than in the part facing the glandular cavity. Sometimes only the nuclear membrane gives a positive reaction in the form of a line or a dotted line, but on the other hand, the cytoplasm gives no appreciable reaction, and often includes irregular shaped areas which show an extremely strong activity. / Rarely there are a few cells, whose nuclei show a copious activity throughout their whole areas and the portion of whose cytoplasm surrounding nuclei also give an intense activity. The peripheral parts of smooth muscle cells and the cytoplasm of the glandular cells near the tunica propria show the most intense activity. The second strongest activity in the cytoplasm is seen in the part facing the glandular cavity and in the part surrounding the vacuoles. Sometimes two different types of cells can be observed. The cell of the first type does not reach the glandular cavity and shows an intense activity, and the nucleus of this cell also gives a strong activity. The cytoplasm of the cell of another type reaches the glandular cavity showing a weak activity, and the nucleus of this cell also gives a weak activity. These results are quite different from those of the acid phosphatase reaction. In the alkaline phosphatase reaction, it may be stated that the activity is stronger in the basal cells than in the superficial cells, following I to's classification. Occasionally and unsystematically large granules are found in the cytoplasm, and they show a copious activity. The nature of these granules is unknown.

b. Eindings in the case with pilocarpine injection (Fig. 4).

The pilocarpine injection results a general intensification of the enzyme activity in the sweat gland; especially markedly in the tunica propria and in the smooth muscle fibers. The cytoplasm and the nucleus of the basal cell show a stronger activity than in the case without the administration of pilocarpine (Fig. 12). 


\section{Protein}

a. Findings in the case without pilocarpine injection.

The stainability is deepest in the smooth muscle fibers and diminishes in the following order, in the tunica propria and in the cytoplasm of the glandular cells. The stainability of the cytoplasm of the glandular cells is irregular. Even among the cells, which face the glandular cavity, some are stained and the other are not stained. Similarly, among the cells, which do not reach the glandular cavity, some are coloured and the others are not. The stainability of nuclei is also irregular. Some of them are stained deeply and the other are faintly coloured. The nucleoli are stained intensely. Therefore it is impossible to distinguish the superficial cells from the basal cells by the difference of the intensity of the stainability, This staining method can not show mitochondria or secretory vacuoles, but can demonstrate the intercellular secretory canalicules very clearly. These intercellular secretory canalicules do not exist between the superficial cells, but are found between the cells, which are situated in the basal side and do not reach the glandular cavity. This result coincides with the picture in cat material, which was described by T s u ka go $\mathrm{sh}$,

b. Findings after the pilocarpine injection.

By means of the protein detecting method it is impossible to find any difference between the pictures of the glandular cells obtainet with or without the administration of pilocarpine.

\section{Glycogen}

a. Findings in the case without the pilocarpine injection (Fig. 7)

The tunica propria is stained most deeply, but the stained substance is not glycogen. The second strongest stain is seen in the cytoplasm of the glandular cells, but on the other hand the nucleus is not stained at all except the nuclear membrane. The nucleoli and the intranuclear granules are not stained. The cytoplasm of the glandular cells is stained irregularly, and includes deeply coloured portions here and there. The differentiation between the superficial cells and the basal cells, is next to impossible. Within the cytoplasm small granules are seen in places. The majority of these 'granules are localized in the region near the glandular cavity, and the rest of them are found in groups in the perinuclear or basal regions. Occasionally large deeply stained granules are demonstrated around the intercellular canalicules.

b. Findings in the case with the pilocarpine injection (Fig. 8)

Generally the amount of glycogen decreases markedly comparing 
with that in the case without the pilocarpine-injection. The cytoplasmic and the perinuclear glycogen is usually stained less distinctly than in the case without the injection. The cytoplasmic granules, which are found in the portions neighbouring the glandular cavity and in the perinuclear regions, have decreased. Within the cytoplasm of the basal cells, which are in contact with the tunica propria, no trace of glycogen is demonstrated, and small areas lacking graunles are seen in places. The deeply stained large granules, however, are still present, and are generally rather increased comparing with the result in the case without the injection. The tunica propria and the stmooth muscle fibers show no remarkable change. (Fig. 13)

5. Iron hematoxylin stain

a. Findings in the case without the pilocarpine injection (Fig. 9)

The tunica propria gives no appreciable stain. Though the outlines of each cells are mostly not clearly seen, the darkstained cells neighbouring the glandular cavity and the lightstained cells being in contact with the basal layer are clearly distinguished. It is similar to the result reported by I to on the human eccrine sweat gland and the result described by $\mathrm{Tsukagoshi}$ on the cat eccrine sweat gland. The former cells of these two types are cylindrical and surround the glandular cavity with their free faces, and their basal ends are in contact with the tunica propria. The latter cells do not reach the glandular cavity and fasten themselves to the tunica propria. It o's secretory granules are seen abundantly around the nucleus of the superficial cell, but found scarcely in the basal cell, being demonstrated only in the portion above its nucleus.

The superficial cell has copious secretory vacuoles within its socalled secretory granules, but the basal cell has no secretory vacuoles. As reported by Tsukagoshi, the cells of these two types both contain in their cytoplasm stainable granules, which are somewhat larger than mitochondria.

b. Findings in the case with the pilocarpine injection (Fig. 10)

Though no general remarkable change is observable, the small granules in the superficial cells have much increased. In the basal cells, on the other hand, the small granules are not seen whereas the large granules have increased.

\section{Discussion}

a) Mitochondria

The mitochondria of the eccrine sweat gland have been studied 
on human material by I t o (1949), on cat material by $\mathrm{T} \mathrm{a} \mathrm{ka} \mathrm{gi} \mathrm{et}$ Hor i (1941) and by Tsukagoshi (1949). According to these investigators, copious mitochondria are demonstrated in the eccrine sweat gland cells stained with iron-hematoxylin, and their shape is in wide variety from threads to granules, and the thread-like mitochondria run from the base of the cell towards the glandular cavity. Besides, Tsukagoshi reported that in his iron-hematoxylin stained sections granules of another type are demonstrated, which are somewhat larger than the granular mitochondria. The more these larger granules are present, the less are the mitochondria. From this result he as sumed that these granules are the secretory granules produced from mitochondria. Moreover he found that in the light-coloured cells, which correspond to the basal cells, mitochondria are hardly seen but in the portions of the cytoplasm distal from the nuclei a few secretory granules are brought forth being stained deeply by iron-hematoxylin. In the observation of the acid phosphatase activity the author found in the superficial cells abundant granules, which are considered to be mitochondria, and in the basal cells a few granules and threads, which are also considered as mitochondria.

I to et $\mathrm{O}$ t a (1949) ascertained in their study on glycogen in the human eccrine sweat gland, that the superficial cells do not contain glycogen and the basal cells alone contain it, and that it is possible to distinguish these cells from each other by the difference of glycogen contents. Although Tsukagoshi reported that in the cat eccrine sweat gland glycogen does not form granules but distribute diffusely.

The author, however, found that there are copious granules both in the superficial and the basal cells, though their distribution is irregular. Therefore, it has been known that it is immpossible to distinguish these cells from each other by the difference of the amount of glycogen contents. Tsukagoshi described that there are irregularformed vacuoles in the basal part of the glandular cells, and an intense glycogen reaction is observed in these vacuoles, especially along their walls. The author, however, did not recognize these pictures. The disagreement of these results comes probably from the difference of the methods employed.

In the observations by means of the iron-hematoxylin stain, as well as in the study with the acid phosphatase reaction, the author demonstrated copious secretory granules in the superficial cells and a few of them in the basal cells. I to and his collaborator reported that in the human eccrine sweat gland there is the formation of the 
secretory vacuoles from the secretory granules, and it lacks in the basal cells.

Tsukagoshi, on the other hand, stated, that, if examined more precisely, the vacuoles are found also in the basal cells among the secretory granules, and the size of these vacuoles is nearly the same as that of the granules.

The author, agreeing with Ito's findinge, found the secretory granules and the secretory vacuoles abundantly in the superficial cells, but could not find any secretory vacuŭles in the basal cells. In all the observations carried out with these three staining methods, the author could show the stained granules, which are somewhat larger than the granular mitochondria, as having been observed by $\mathrm{T} \mathrm{suk} \mathrm{a-}$ gosh i in his iron-hematoxylin stained sections.

Mitochondria can not be demonstrated by the alkaline phosphatase reaction nor by the protein staining procedures.

b) The intercellular secretory canalicules (Fig. 9, Fig. 6., Fig. 8. and Fig. 13).

According to the study by Ito and Tsukagoshi, the secretory canalicules are not present in the part of the gland where the glandular cells are arranged in a single layer, but localized only in the part, where the glandular cells are arranged in double layers. There are no canalicules among the cells which surround the glandular cavity, but they are found along the cells, which are situated on the basal side and do not reach the glandular cavity. The canalicules run through the space between the former cells and join the glandular cavity. Following these investigators opinion, these findings show the proof that the basal cells have the secretory function.

In the present study by means of the staining procedures for protein and glycogen and the iron-hematoxylin stain method, the author demonstrated the secretory canalicules clearly along the basal cells, agreeing with the results reported by Ito and Tsukagos hi.

c) The difference between the results obtained from the cases with and without the administration of the pilocarpine injection.

$\mathrm{Y}$ u y a m a (1935) stated that during the perspiration caused by the administration of heat or the pilocarpine injection, the glycogen contents of the human eccrine sweat gland diminishes gradually and sometimes to totally none. Schelley and Mescon (1952) compared the non-functioning and the perspirating glandular cells in their histochemical study of the human eccrine sweat gland. The perspiration was caused by previous warming or by the administration of the 
pilocarpine injection. These investigators observed the gland employing the histochemical methods for acid phosphatase, alkaline phosphatase and glycogen.

Their results demonstrated that the acid phosphatase and the alkaline phosphatase activities do not change at all with time after the perspiration. On the other hand, the glycogen contents of the glandular cells decrease or sometimes disappear, as it is demonstrated by Yu y a ma.

In the present experiment, the author found: in five minutes after the perspiration that the acid phosphatase activity is generally intensified to some extent, and the alkaline phosphatase activity also generally increases markedly. The acid phosphatase reaction demonstrates the increase of the secretory granules both in the superficial cells and in the basal cells. The intensification of the alkaline phosphatase activity is remarkable in the tunica propria, the smooth muscle fibers and the basal cells (Fig. 11 and Fig. 12).

In five minutes after the perspiration, the glycogen contents in the cytoplasm decrease and the granules disappear, though the decrease is not so distinct as observed in Shelley and Mescon's experiment (in 45 minutes after the perspiration).

The disappearance of glycogen is distinctly recognized particularly in the part, which is considered as the basal cells.

These changes of the basal cells, demonstrated by the staining techniques for the alkaline phosphatase reaction and glycogen, show that the basal cells have a secretory function (Fig. 13).

The picture stained by the iron-hematoxylin procedure is similar to what is visual by the acid phosphatase, technique, showing the increase of the secretory granules and the appearance of the gross granules in the basal cells (Fig. 14).

\section{Summary}

The author observed cytochemically the effect of the pilocarpine administration of the plantar sweat gland of the cat, comparing the results obtained in the cases with and without the pilocarpine injection, by applying the detecting procedures for acid phosphatase, alkaline phosphatase, protein and glycogen and the iron-hematoxylin technique.

The presence of the cells of the two different types, the superficial and the basal cells, which was described by It 0 and his collaborator on the human eccrine sweat glands, has been ascertained cytochemically 
by the author also on the cat eccrine sweat glands. These cells can be distinguished cytochemically by the difference of the intensities of the acid phosphatase activity. The differentiation of these cells from each other is not appreciably distinct by the alkaline phosphatase technique, and it is impossible by the protein and the glycogen stain procedures.

After the administration of pilocarpine by injection, the most distinct changes are seen in the alkaline phosphatase activity and the glycogen contents, and the acid phosphatase activity and the stain reaction by the iron-hematoxylin technique change moderately. Generally these changes are remarkable in the basal cells.

From these findings the author concludes that the basal cells have also a secretory function as well as the superficial cells, and play a certain roll in the perspiration caused by the pilocarpine injection.

The stainability of protein is not influenced by the pilocarpine perspiration, showing no change in the cytomorphological features.

\section{Acknowledgement}

The author expresses his most cordial thanks to Dr. Prof. T. $\mathrm{T}$ a $\mathrm{nig} \mathrm{u} \mathrm{chi}$ for his invaluable guidance. This work has been supported in part by a grants-in-aid from The Education Ministry given to Dr. M. Ih n u ma.

\section{References}

Go mor i, G. 1952. Microscopic histochemistry. The Univ. of Chicago Press, Chicago. I to, T. 1943. Cytological studies of eccrine sweat gland in human skin. Biol. and Med., 6: 80 (in Japanes:).

I to, T. 1949. Histology and cytology of sweat gland. Igaku-no-shinpo, 6: 106, (in Japanese).

I to, T. and Oois hi, K. 1947. Cytolozical study on the human eccrine sweat gland with special referance to its apocrine secretion. Kaibo. Z., 23: 48 (in Japanese).

It $0, T$. and $O t a, T$. 1949. On the glycogen in sweat gland. Seitai-no-Kagaku, 1: 146 (in Japanese).

I to, T. and I w a hige, K. 1951. Zytologische Untersuchungen uber die ekkrinen Schweissdritsen in menschlicher Achselhaut mit besonderer Berücksichtigung der apokrinen Sekretion derselben. Fol. anat. jap., 23 : 174.

Shelley, W.D. and Mescon, H. 1952. Histochemical demonstration of secretory activity in human eccrine sweat glands. J. Invest. Dermatol. 18 : 289.

Ta kagi, S. and Hori, S. 1941. The fine structure of sweat gland in cat. NipponSeirigaku-Zasshi, $6: 667$, (in Japanese).

Tsukagoshi, N. 1951. Zur Zytologie der ekkrinen Sçhweissdrüsen der Tiere mit besonderer Berücksichtigung des Vorkommens der zwei Arten Drüsenzellen und ihrer apokrinen Sekretion. Arch. hist. Jap., 2: 481 (in Japanese). 


\section{Explanation of Plates}

Abbreviations :
A.
B. .............................. Basal cell
C. .............................. Secretoy granules
D. ............................. Gross granules

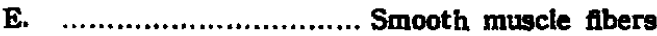
F. ............................. Tunlca propria
G. ........................... Intercellular secretory canalicules

Fig. 1. Acid phosphatase reaction in the case without the pilocarpine injection.

It is clearly seen that the gland is composed of cells of two different types, $A$ and $B$. The activity is far stronger in $A$ than in $B$. Granular or threadlike mitochondria are seen more abundantly in $\mathbf{A}$ than in $\mathbf{B}$.

Fig. 2. Acid phosphatase reaction in the case after the pilocarpine injection.

Activities in A and B are both intensified, particularly much in B. Granules have increased in B. Gross granules in A also increased.

Fig. 3. Alkaline phosphatase reaction in the case without the pilocarpine injection.

The activity is iutense in $E$ and $F$. The activity of the glandular cells comes second. Though the activity is generally weaker in $A$ than in $B$, it is difficult to distinguish $A$ and $B$ from each other by the difference of intensities of the activity. The nature of the granules, seen in places, is unknown.

Fig. 4. Alkaline phosphatase reaction in the case after the pilocarpine injection.

A general intensification of the activity is seen comparing with seen in Fig. 3. F and $E$ show a remarkable intensification. The activity in the cytoplasm and the nucleus of $B$ is also much intensified. $A$ shows no appreciable change.

Fig. 5. Protein reaction in the case without the pilocarpine injection.

It is impossible to distinguish $A$ and $B$ from each other. $G$ is clearly demonstrated. They are not seen among $\mathbf{A}$ cells, but run along $B$ cells, which do not reach the glandular cavity.

Fig. 6. Protein reaction in the case after the administration of pilocarpine.

No remarkable change is observable in the findings, comparing with those seen in Fig. 5.

Fig. 7. Glycogen stain, in the case without the pilocarpine injection.

The stained substance in $F$ and $E$ is not glycogen. Two different types of cells, $A$ and $B$, are dificult to distinguish from each other by the stainability of glycogen. Small granules are present distributing themselves irregularly. Around G. deeply stained gross granules, D, are seen.

f A general decrease of glycogen is clearly observed, comparing with the result seen in Fig. 7. The most prominent decrease of glycogen occurs in part, which is neighbouring $F$ and is considered as the cytoplasm of $B$. C decreases generally. D gives an unchanged positive reaction.

Fiz. 9. The iron-hematoxylin stain, in the case without the pilocarpine injection.

A and $B$ are clearly visible. The cytoplasm of A contains copious secretory 
granules, C. D are seen in places.

Fig. 10. The iron-hematoxylin stain, in the case after the administration of pilocarpine. $D$ have increased in the cytoplasm of $B$.

Fig. 11. After the pilocarpine injection.

The activity is intensified in $\mathbf{A}$ and $B$. The granules in $B$ particularly increased markedly.

Fig. 12. After the pilocarpine injection.

The activity is greatly intensified in $\mathbf{E}, \mathrm{F}$, and $\mathrm{B}$.

Fig. 13. After the pilocarpine injection.

A general decrease of glycogen is observed.

A remarkable diminishing of glycogen is seen in the part, which is considered as being the cytoplasm of $B$.

Fig. 14. After the pilocarpine injection.

The gross granules, D, have increased in $B$. 
Plate I
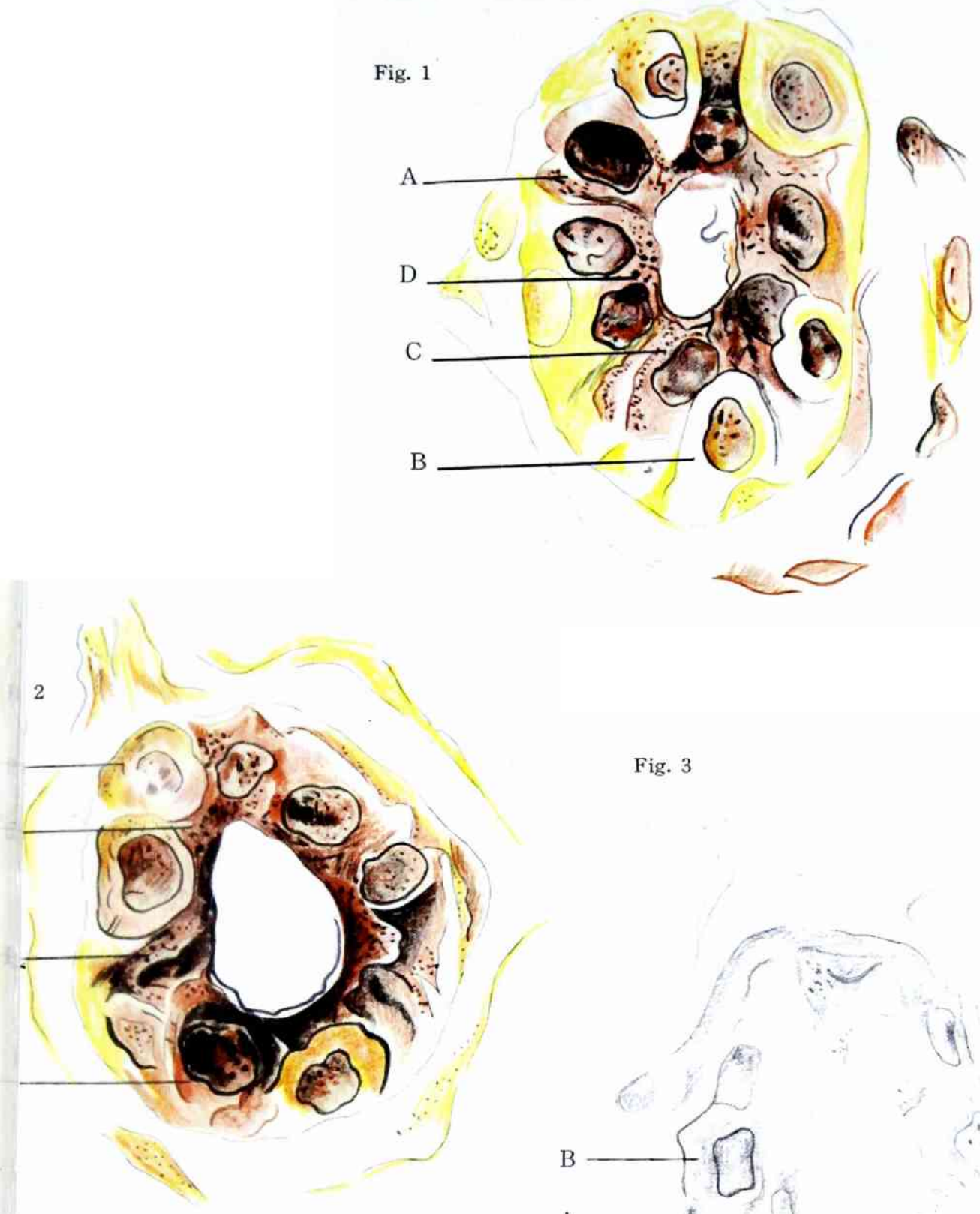

Fig. 3

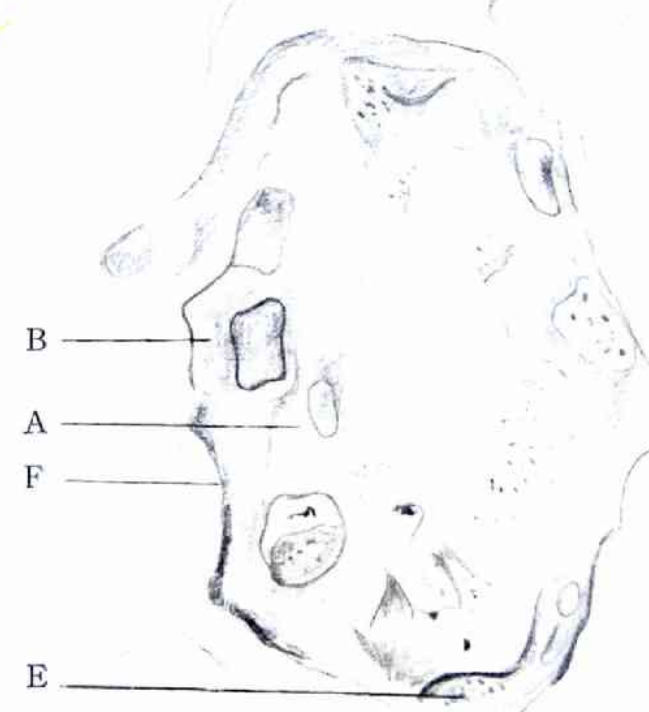

j. Kamamura 
Plate II

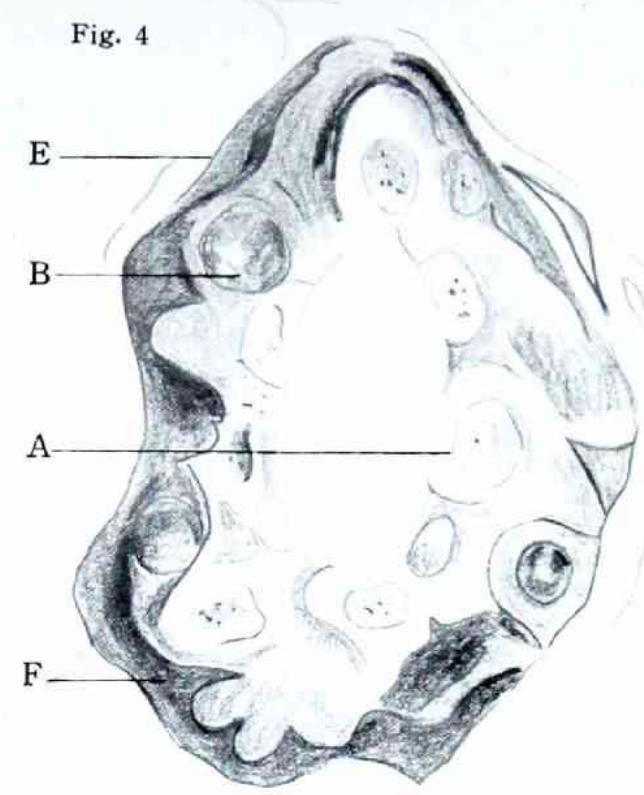

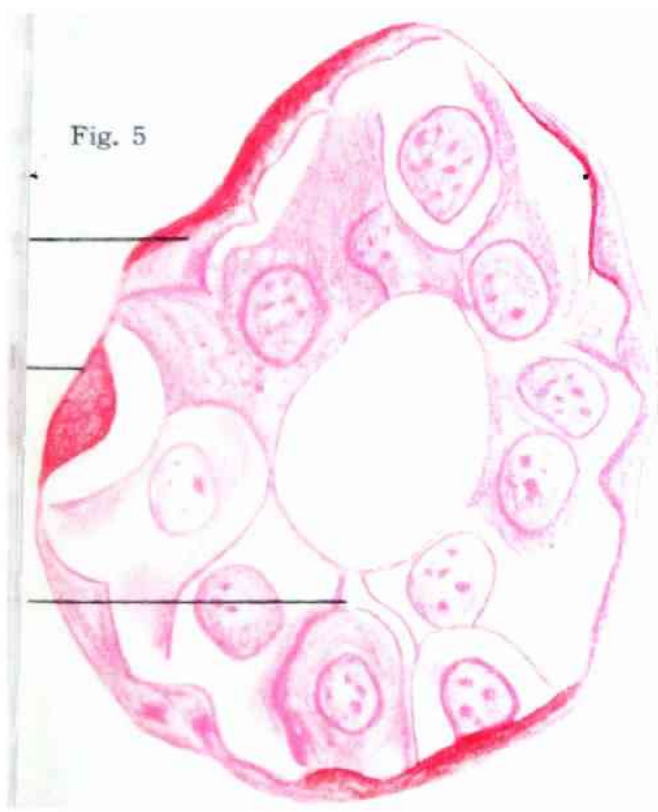

Kamamura

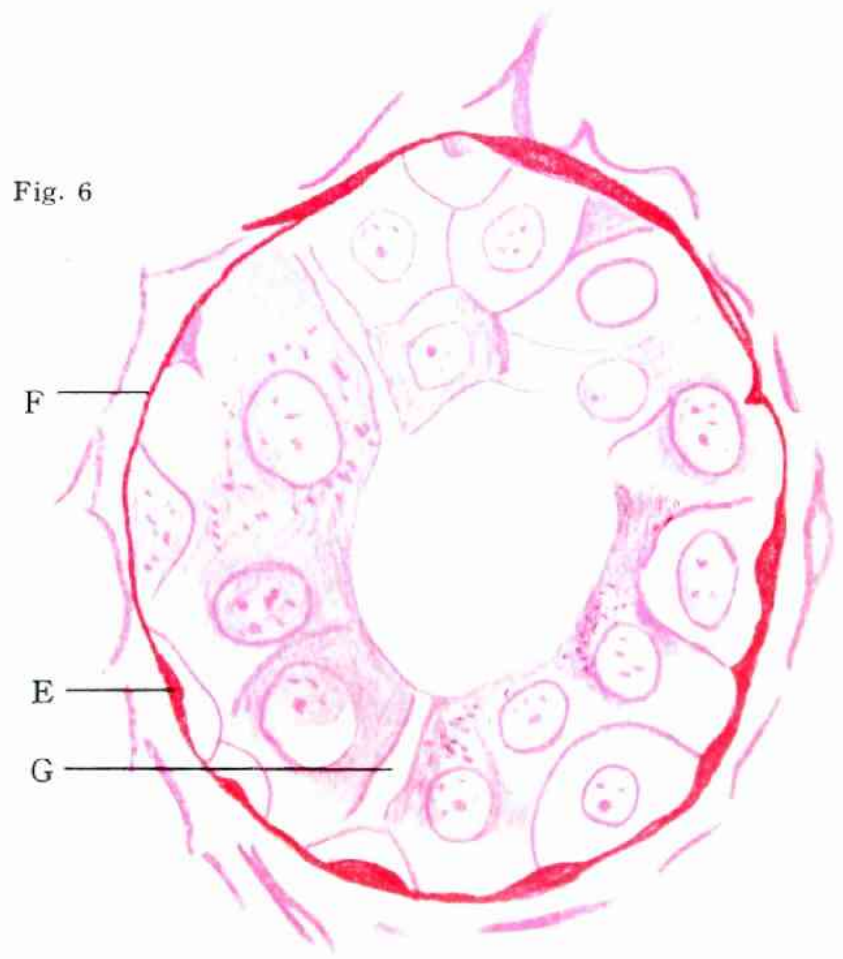


Plate III

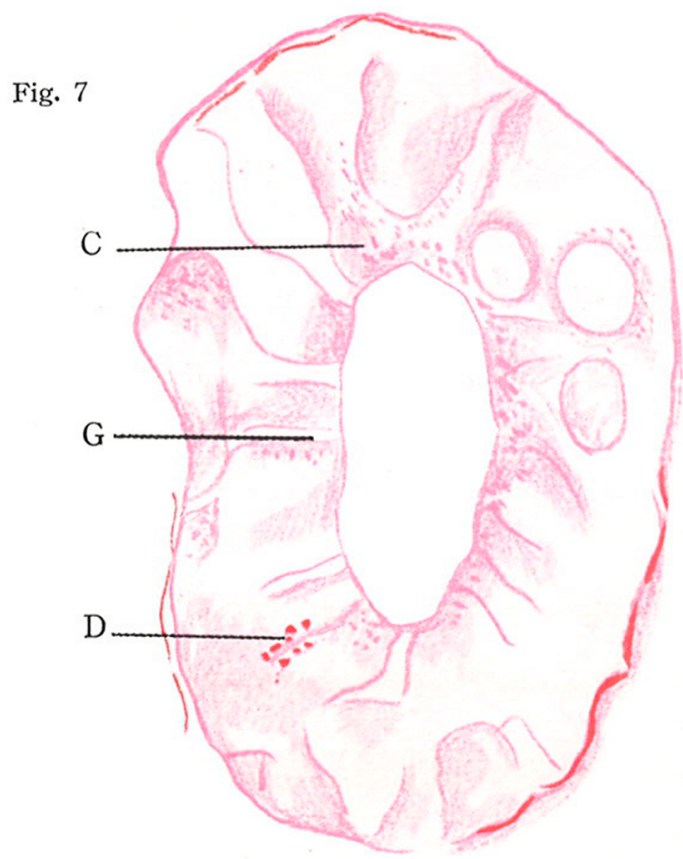

Fig. 9

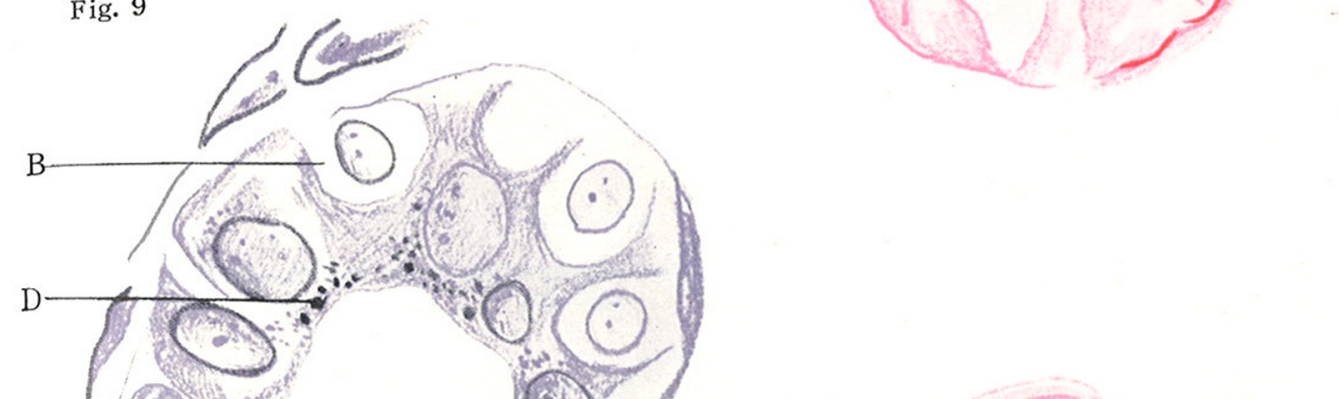

Fig. 8
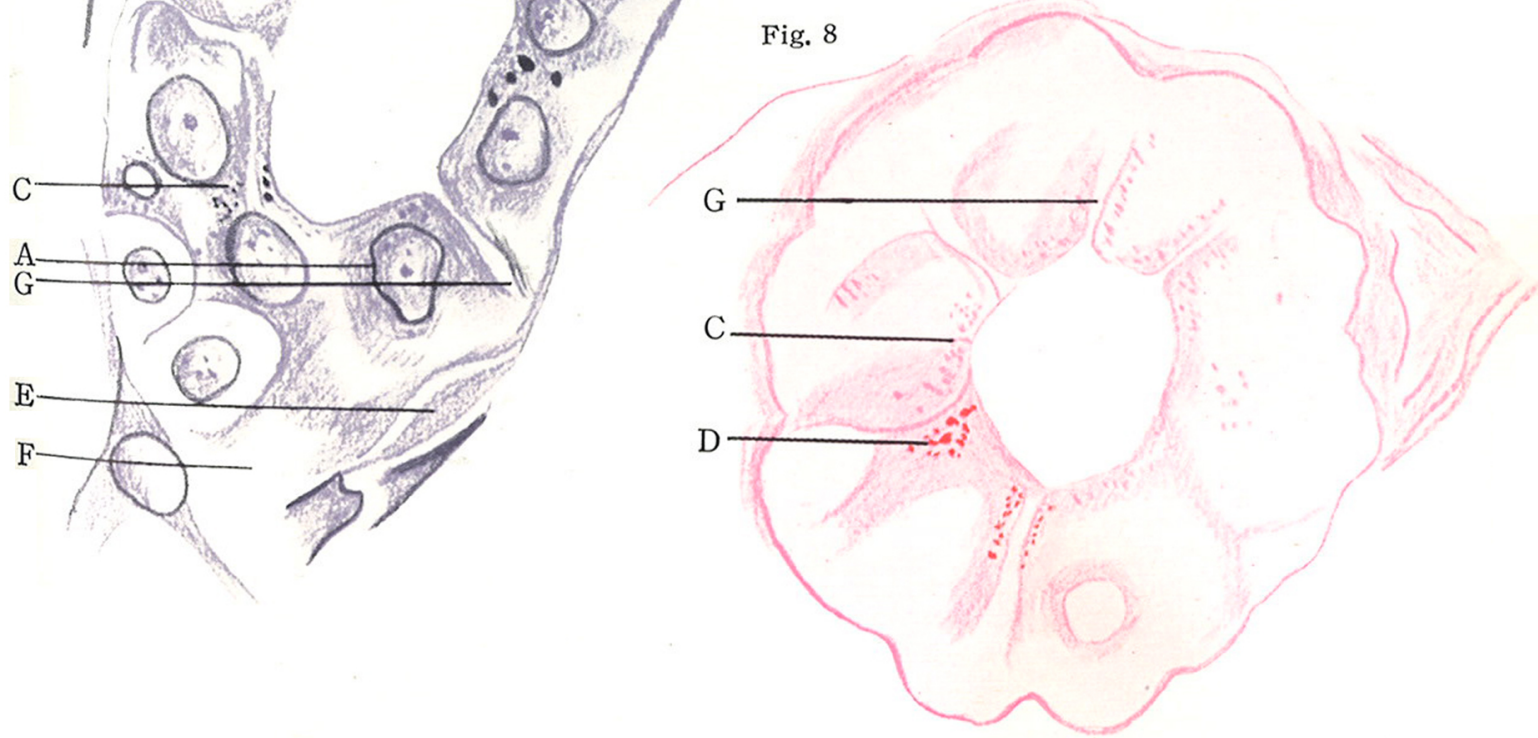

S. Kamamura 

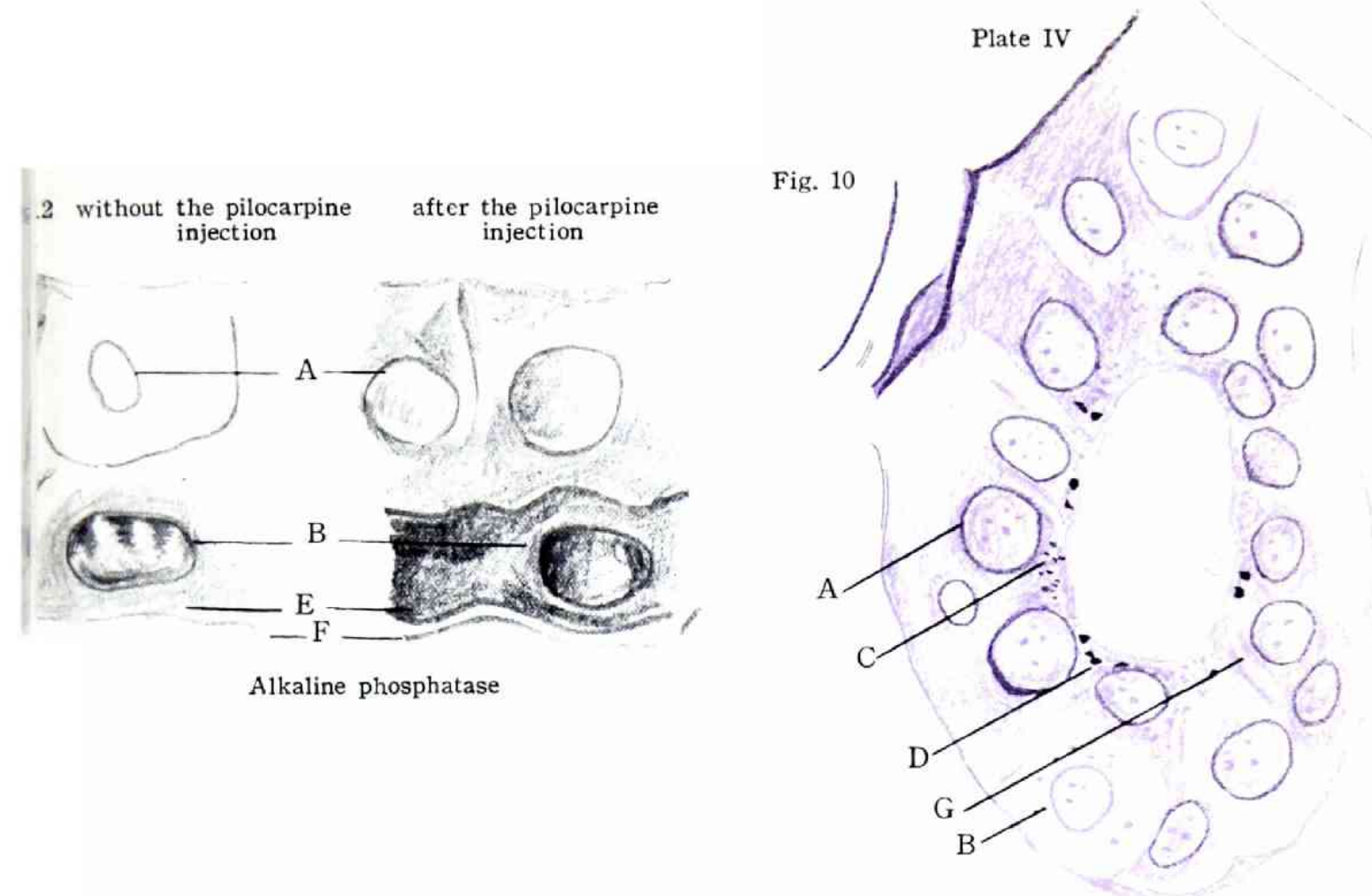

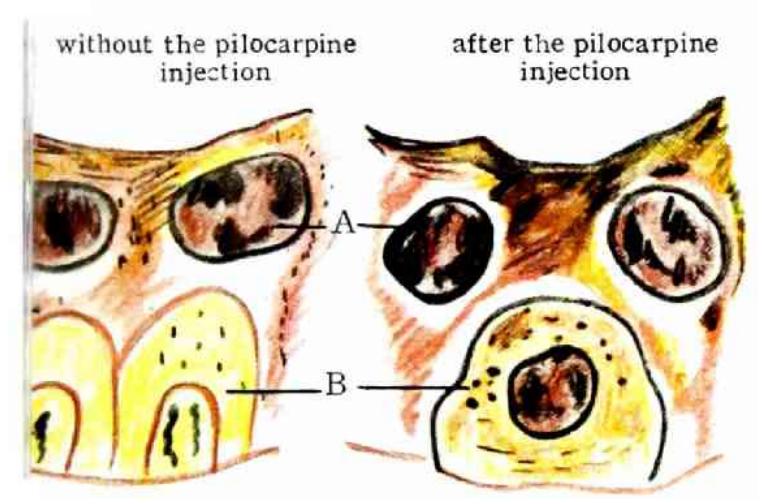

Acid phosphatase
Fig. 13 without the pilocarpine after the piloçarpine injection

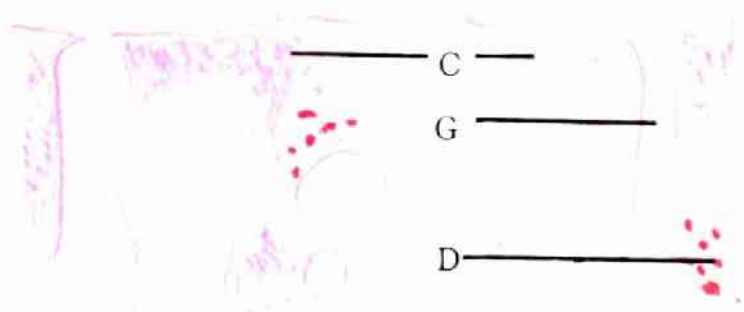

Glycogen stain

Fig. 14 without the pilocarpine after the pilocarpine injection injection

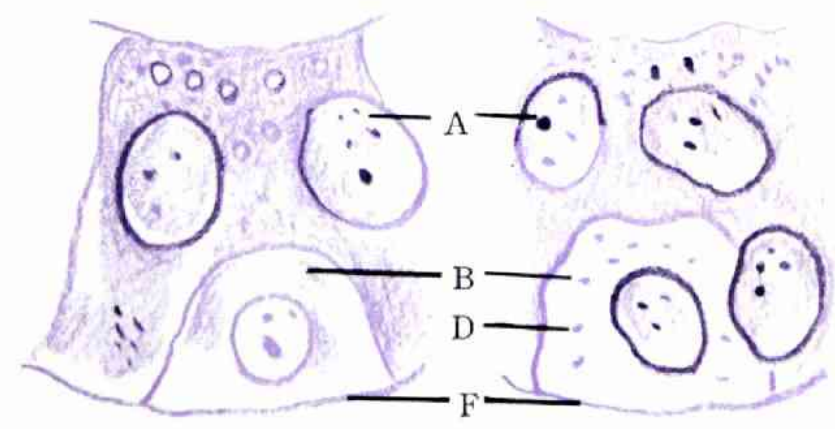

